\title{
Genomic prediction in an outcrossing and autotetraploid fruit crop: lessons from blueberry breeding
}

\author{
Luís Felipe V. Ferrão ${ }^{1}$, Rodrigo R. Amadeu ${ }^{1}$, Juliana Benevenuto ${ }^{1}$, Ivone de Bem Oliveira ${ }^{2}$, \\ Patricio R. Munoz ${ }^{1 *}$ \\ ${ }^{1}$ Horticultural Sciences Department, University of Florida, Gainesville, FL, USA \\ ${ }^{2}$ Hortifrut North America, Inc., Estero, FL 33928, USA \\ Corresponding Author p.munoz@ufl.edu
}

\begin{abstract}
Blueberry (Vaccinium corymbosum and hybrids) is a specialty crop, with expanding production and consumption worldwide. The blueberry breeding program at the University of Florida (UF) has greatly contributed to the expansion of production areas by developing low-chilling cultivars better adapted to subtropical and Mediterranean climates of the globe. The breeding program has historically focused on phenotypic recurrent selection. As an autopolyploid, outcrossing, perennial, long juvenile phase crop, blueberry's breeding cycles are costly and time-consuming, which results in low genetic gains per unit of time. Motivated by the application of molecular markers for a more accurate selection in early stages of breeding, we performed pioneering genomic prediction studies and optimization for implementation in the blueberry breeding program. We have also addressed some complexities of sequence-based genotyping and model parametrization for an autopolyploid crop, providing empirical contributions that can be extended to other polyploid species. We herein revisited some of our previous genomic prediction studies and described the current achievements in the crop. In this paper, our contribution for genomic prediction in an autotetraploid crop is three-fold: i) summarize previous results on the relevance of model parametrizations, such as diploid or polyploid methods, and inclusion of dominance effects; ii) assess the importance of sequence depth of coverage and genotype dosage calling steps; iii) demonstrate the real impact of genomic selection on leveraging breeding decisions by using an independent validation set. Altogether, we propose a strategy for the use of genomic selection in blueberry, with potential to be applied to other polyploid species of a similar background.
\end{abstract}

\section{Introduction}

Blueberry (Vaccinium corymbosum and hybrids) is recognized worldwide for its health benefits due to the high content and diversity of polyphenolic compounds (KALT et al., 2020). Such health-related attributes has resulted in an increased demand for blueberries, as it has become a crop with one of the highest production trends, with an increase of $142 \%$ of its production in the last 10 years (FAOSTAT, 2021). In this sense, the blueberry breeding program at the University of Florida (UF) has had a major contribution in the expansion of production areas. Starting in the 1950's, the UF blueberry breeding program led pioneering hybridizations between high-quality US northern adapted species (Vaccinium corymbosum) and endemic US southern species (e.g., Vaccinium darrowii), selecting for low-chill requirements to break dormancy of flower buds (SHARPE and ShERMAN, 1971; LyRENE, 2000). The resulting breeding material and cultivars,

\footnotetext{
Keywords: Feal validation, genomic selection, genotyping by sequencing, sequencing depth, allele dosage, plant breeding, molecular marker, fruit quality

Abbreviations: : UF (University of Florida), GEBV (genomic Estimated breeding value), GWAS (genome-wide association studies (GWAS), QTL (quantitative trait loci), EBLUE (empirical best linear unbiased estimates), SNP (single nucleotide polymorphisms), (GxE) genotype by environment interaction, MAS (marker-assisted selection)
} 
known as southern highbush blueberries, established a new industry in Florida and in other warmer regions worldwide, allowing a year-round supply of fresh blueberries for the global market.

Historically, the UF program, like many others, used phenotypic recurrent selection with visual assessment of plants to select both new parents for crossing and clones to for commercial testing (CELlON et al., 2018). Despite the success of the industry and the release of many cultivars in recent decades, the use of conventional methods results in low genetic gains per unit of time. Moreover, the autopolyploid nature of the crop, long juvenile phase, multi-year evaluations, large experimental areas, and the high sensibility to inbreeding depression makes phenotypic selection costly and time-consuming. Remarkably, it can take up to 12 years to release a new cultivar using conventional tools (LYRENE, 2005). As DNA sequencing costs continue to decrease, genomics-based markers present an opportunity to accelerate the breeding process by achieving more accurate selection during earlier breeding stages. Thus, the UF blueberry breeding program has been leading innovative genomics studies and procedures to fill two primary gaps in the blueberry breeding literature: understanding the genetic architecture of complex traits via genome-wide association studies (GWAS) and quantitative trait loci (QTL) mapping; and, at the practical level, performing phenotypic prediction based on molecular markers, a methodology popularly referred to as genomic selection.

GWAS and QTL mapping are both tools for providing biological elucidation of the genetic architecture, in which molecular markers spanning the entire genome are statistically tested for associations with phenotypes (PRITCHARD et al., 2000). While QTL analyses are usually performed using structured populations, GWAS increases the mapping resolution by making use of populations with low levels of linkage disequilibrium considering a deep history of recombination events. In blueberry, we recently detected candidate genomic regions and markers associated with different fruit quality traits (FERRÃo et al., 2018) and flavor-related volatiles (FERRÃo et al., 2020) via GWAS investigations; and we built a high-density linkage map and detected QTL associated to berry firmness (CAPPAI et al., 2020a). In counterpart, genomic selection aims to predict breeding values by using all genome-wide markers simultaneously (MEUWISSEN et al., 2001). The underlying rationale is that, whenever the marker density is high enough, most QTL's will be in linkage disequilibrium with some markers. Therefore, the estimated effect of all markers will lead to accurate predictions of the genetic merit for a complex trait. We have recently shown the potential of genomic selection in blueberry breeding under distinct modeling scenarios (DE BEM OliveIRA et al., 2019, 2020; Amadeu et al., 2020; Zingaretti et al., 2020).

The autopolyploid nature of blueberry $(2 \mathrm{n}=4 \mathrm{X}=48)$ imposes additional challenges for analysis and interpretation of genetic data. Autopolyploids possess genomes with multiple sets of homologous chromosomes, resulting in non-preferential pairing and potential polysomic inheritance during meiosis. Given the presence of higher allele dosage (i.e., the number of copies of each allele at a particular locus), a higher number of genotypic classes are possible (Gallais, 2003; Garcia et al., 2013; Dufresne et al., 2014). The inclusion of allelic dosage information on genomic selection models could imply in a more accurate estimation of breeding values by considering the additive effect of multiple copies of the same allele and the potential inheritance of dominance effects. However, accurate allele dosage calling on polyploids depends on higher depth of coverage which can increase genotyping costs when using sequence-based genotyping platforms (GERARD et al., 2018; CARUAna et al., 2019). After performing foundational studies on the importance of polyploid models, inclusion of non-additive effects, and sequencing depth on allele dosage parameterizations, the UF blueberry breeding program is now on track to overcome the barrier of a simple promise to guide breeding decisions, to make genomic selection a reality.

Motivated by the potential to use genomic selection to reshape traditional blueberry breeding, we herein revisited some of our previous studies and described the current achievements in blueberry. Thus, our contributions in this paper are three-fold: (i) summarize previous results on the relevance of model parametrizations, such as diploid or polyploid methods, and inclusion of additive and non-additive gene actions for prediction; (ii) assess the importance of accurate dosage estimation for genomic prediction under low and high sequencing depth scenarios; (iii) demonstrate the realized impact of genomic selection over breeding cycles by using an independent validation set. Altogether, we anticipate elucidating challenges and directions for future studies in blueberry and relating our findings to other polyploid and fruit species with a similar breeding background. 


\section{Material and Methods}

\subsection{Populations and phenotypic data}

The southern highbush blueberry populations used in this study were generated as part of the breeding program at the University of Florida. Two phenotypic datasets, referred as calibration set and testing set, were used with different purposes. The calibration set is comprised of a large breeding population already in use and is described in previous studies (FERRÃo et al., 2018; DE BEM Oliveira et al., 2019). Briefly, it consists of 1,834 individuals originating from 117 biparental crosses using 146 distinct parents. All phenotypic evaluations were conducted on ripe fruits collected from the beginning of April to mid-May. Fruit firmness ( $\mathrm{g}^{*} \mathrm{~mm}-1$ of compression force), size $(\mathrm{mm})$, and weight $(\mathrm{g})$ were evaluated over two seasons (2014 and 2015), while soluble solid (Brix) was evaluated only in 2015. Given the large representative population, all genomic prediction models reported in this study were calibrated using this dataset. The empirical best linear unbiased estimates (eBLUEs) were estimated for each genotype based on a linear model, where genotype and year were considered fixed effects, as described by AMADEU et al. (2020). Hereafter, the eBLUEs for each trait were considered as our response variable in the genomic prediction analyses.

The testing set was used for real validation in genomic prediction analyses. It comprises 280 advanced selections not originally included in the calibration set. These genotypes represent materials in the final evaluation stages in the breeding program. Hence, they were evaluated over several years in different locations throughout the state of Florida. As these phenotypes were collected from plants in different physiological phases and multiple environments, we adjusted the phenotypes using a linear model including separate fixed effects for year, location, and plant age. The eBLUEs of each genotype per trait were used as the phenotypic value in subsequent genomic prediction analyses. All phenotypic analyses were carried out using the ASREML-R software (BUTLER et al., 2009).

\subsection{Genotyping}

The calibration set was genotyped using the "Capture-Seq" approach as described in BenEvenuto et al. (2019). The genotyping of the testing set was also performed using "Capture-Seq" considering 10,000 biotinylated probes of 120-mer at RAPiD Genomics (Gainesville, FL, USA). Sequencing was carried out in the Illumina HiSeq2000 platform using 150 cycle paired-end runs. To ensure that the same group of single nucleotide polymorphisms (SNPs) will be called in both calibration and testing sets, we included the nextgeneration sequence data from both sets under the same SNP calling pipeline. Raw reads were cleaned and trimmed. The remaining reads were aligned using Mosaik v.2.2.3 (LEE et al., 2014) against the largest scaffolds of each of the 12 homoeologous groups of Vaccinium corymbosum cv. 'Draper' genome assembly (Colle et al., 2019). Single nucleotide polymorphisms (SNPs) were called with FreeBayes v.1.3.2 using the 10,000 probe positions as targets (GARRISON and MARTH, 2012). Loci were filtered out applying the following criteria: minimum mapping quality of 10 ; only biallelic locus; maximum missing data of $50 \%$; minor allele frequency of $1 \%$; and minimum and maximum mean sequence depth of 3 and 750 across individuals, respectively. A total of 63,552 SNPs were kept after these filtering steps. Sequencing read counts per allele per individual were extracted from the variant call file using vcftools v.0.1.16 (DANECEK et al., 2011) and subsequently used to investigate some practical questions for the implementation of genomic prediction in polyploids.

Within the calibration set, we first investigated the importance of accurate genotype calling by comparing two strategies: (i) for the ratio method, each genotypic score was computed as the ratio between the alternative and total read depth, as described by SvERRISDótTir et al. (2017) and applied in DE BEM OliveIRA et al. (2019); (ii) for the dosage method, genotypic classes were assigned probabilistically using the updog v.2.1.0 R package considering the "norm model" and prior bias equals zero (GERARD et al., 2018; GERARD and FERRÃo, 2020). Both genotyping methods (ratio and dosage) were compared under scenarios of high sequencing depth (random sampling for mean number of 60 reads - 60x) and low sequencing depth (random sampling for mean number of 6 reads $-6 \mathrm{x}$ ). Specifically, we assumed the sequencing reads of each allele (alternative or reference) for a given marker come from a multinomial distribution, with probability 
equal the number of the reads divided by the total number of reads across all the alleles, markers, and individuals $(\mathrm{N})$. Then, we sampled N/10 reads from this multinomial distribution. We performed this sampling 10 times, and each sampling result was used in a different cross validation fold. To avoid an eventual confounding between the number of markers and the predictive capacity over the four scenarios, we kept the same number of SNPs $(63,552)$ across all scenarios. Therefore, in total, four scenarios were tested: ratio_60x, ratio_6 6 , dosage_60x, and dosage_6 6 .

For the real validation and implementation of genomic selection in the blueberry breeding program, we used the actual read counts to estimate the allele dosage in the calibration and testing sets according to the "norm model" in the updog v.2.1.0 R package (GERARd et al., 2018; Gerard and Ferrão, 2020). The posterior probability modes were used as our genotypic score. After estimating the posterior mean per genotype, we filtered out markers with a proportion of individuals genotyped incorrectly (prop_miss $<10 \%$ ), and markers with estimated bias higher than 0.13 and smaller than 7.38. Missing genotypes were imputed by the mean of each locus. A total of 48,829 SNPs were kept and used in genomic prediction for real validations.

\subsection{Statistical Analyses}

Single-trait linear mixed models were used to predict breeding values using the restricted maximum likelihood approach (REML) as following: $y=\mu+Z u+e$; where $\mathbf{y}$ is a vector of pre-corrected phenotypic records for a particular trait; $\mu$ is the overall mean; $\mathbf{Z}$ is an incidence matrix linking observations in the vector $\mathrm{y}$ to their respective breeding value in the vector $\mathbf{u}$. Normality where assumed for the additive and residual effects, where $u \sim \operatorname{MVN}\left(0, G \sigma_{u}^{2}\right)$ and the residual variance $e \sim M V N\left(0, I \sigma_{e}^{2}\right)$. For the residual, $\mathbf{I}$ is an identity matrix; while and are the genetic and residual variance components. The matrix $\mathbf{G}$ denotes the genomic relationship matrix computed either using the ratio genotypic score or the tetraploid allele dosages with the different sequencing depths as described above. The matrices were estimated in the AGHmatrix v.2.0.0 R package (AMADEU et al., 2016). For the ratio implementation, we used the "ratio" option in the software that compute the relationship as: $G=\frac{Z Z^{\prime}}{h}$, where $\mathbf{Z}$ is the mean-centered matrix of the molecular marker information (ratio values); and $h$ is a scale factor, where $h=\sum_{i=0}^{m} s_{i}^{2}$ and $s_{i}^{2}$ is the variance of the vector $z_{i}$ centered marker vector (for more details, see DE BEM OliveIRA et al. (2019)). For the dosage implementation, we used the additive relationship matrix based on VANRADEN (2008) as described by DE Bem Oliveira et al. (2019). All genomic prediction analyses were carried out using the rrBLUP package (Endelman, 2011).

Predictive performances were accessed for the ratio and dosage methods under high (60x) and low (6x) sequencing depth scenarios using only the calibration set in a 10-fold cross-validation scheme. To this end, the calibration set was randomly divided into 10 groups, where one group was used as a validation test, while the remaining nine groups were used as training. Models were trained in the validation test using the GBLUP approach. For each fold, predictive abilities were estimated using Pearson's correlation between genomic estimated breeding values (GEBVs) and the corresponding eBLUEs. We also evaluated the correspondence between the top 20 group of individuals ranked using dosage_60x and the other scenarios. A post hoc Tukey test (p.value $=.05$ ) was used for intergroup comparisons between the top 20 ranked genotypes.

For the real genomic selection validation over the breeding cycles, we assessed the robustness of our predictive model over different stratification levels: (i) General predictions stand for models trained in the calibration set and predictions carried out in the testing data, in which the target phenotypic values were pre-corrected for year, location, and age fixed effects; (ii) For the across-stages predictions, a group of 114 individuals originally included in the calibration set but not in the testing set, was cloned in 2014 and planted in a commercial condition in a single location - prediction accuracy in this scenario can demonstrate the potential losses when models are trained at earlier stages and used at late stages of selection; (iii) In the stratified predictions, models trained in the calibration set were tested for predictions across four regions in Florida (North-FL, Central-FL, South-FL, and Citra-FL) - in contrast to the general predictions, in this scenario the target phenotypic values were pre-corrected only for the year effect per region. In all scenarios predictive performances were accessed via Person's correlation between predicted and corrected phenotypic values, depending of the validation scenario. 
A summary of all validation scenarios is illustrated in the Figure 1. For the stratified predictions, we complemented the predictive analysis by accessing the importance of genotype-by-environment interaction (GxE) via Analysis of Variance (ANOVA). To this end, we considered 16 genotypes (checks) that were phenotyped over the four regions. We fitted a linear model considering year, genotype, location, and the interaction between genotype and location $(\mathrm{GxE})$ as fixed effects Analysis of Variance were performed in $\mathrm{R}$ (TEAM, 2013) using the native $\operatorname{Im}()$ function.
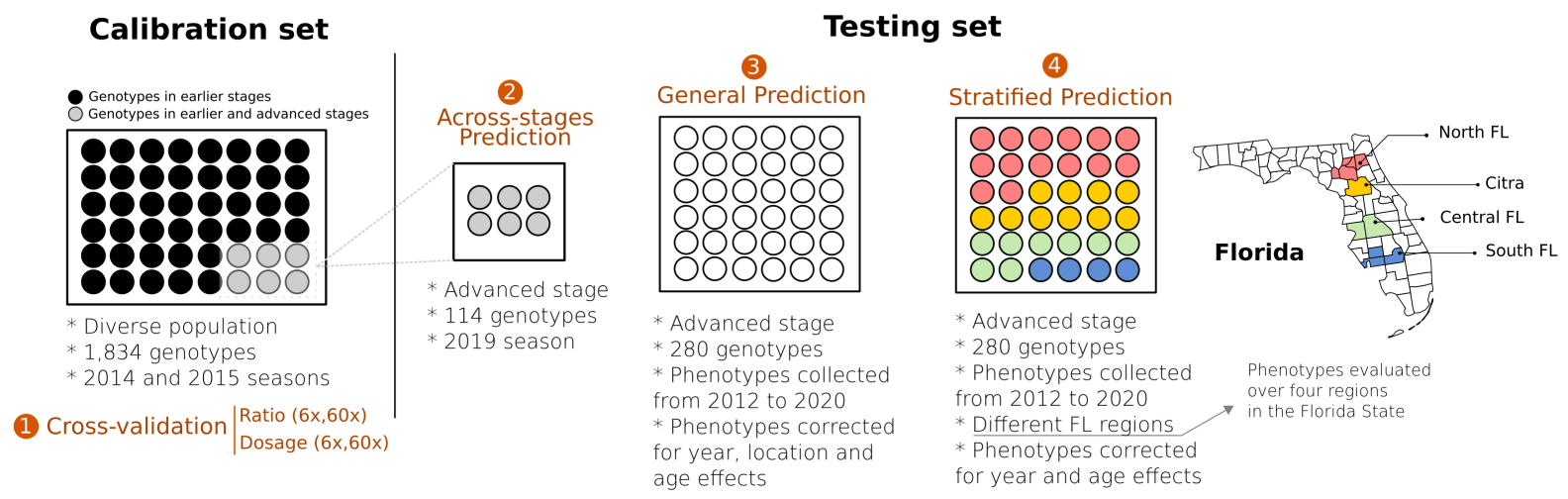

Figure 1: Schematic representation of four validation scenarios tested in blueberries. Calibration set represents a diverse group of genotypes were all genomic selection models where originally trained (DE BEM Oliveira et al., 2019). In the calibration set, using 10-fold cross validation, we tested the relevance of genotyping calling (ratio vs. dosage) under two different sequencing depth (6x and 60x) in scenario 1 of validation. Scenario 2 (across-stages) represents a group of 114 individuals originally presented in the calibration set that were clonally propagated, moved to advanced stage of the breeding program, and phenotyped under commercial field conditions. Scenario 3 (general prediction) represents a group of independent 280 genotypes, evaluated under commercial conditions, in which the phenotypic values of the target individuals were pre-adjusted for the year, location and age effects. Scenario 4 (stratified prediction) is an attempt to perform predictions over four regions of the State of Florida.

\section{Results and Discussion}

In the last two decades, genomic selection has become a reality for many animal and plant breeding programs. Despite the optimism and proved efficacy, its wide implementation is still hindered by investment costs and analytical skills required (HICKeY et al., 2017). With that in mind, the UF blueberry breeding program initiated genomic studies on a large scale in 2013. To begin, we worked closely with genotyping companies to design customized genotyping platforms; we phenotyped and genotyped a large and diverse blueberry breeding population; we increased our computational resources; and finally, we adapted our breeding framework to incorporate genomics. During this process, the implementation of genomic selection in polyploid and outcrossing species proved to be challenging, particularly regarding the intrinsic biological complexities and the availability of genomic and computational tools (MACKAY et al., 2019). In blueberry, for example, a high-quality genome assembly became available only in 2019 (COLLE et al., 2019). About half of the capture-seq genotyping probes that were originally developed based on a draft genome assembly were discarded afterwards based on the high-quality genome, without compromising genetic association and genomic prediction analyses (BENEvEnUto et al., 2019). We also explored additional optimizations to reduce costs, regarding the number of individuals per family, number of markers, and sequencing depth (DE BEM Oliveira et al., 2020). Moreover, new genomics methods and tools have been developed in the last decade for the polyploid community, including allele dosage estimation, haplotype reconstruction, and the use of 
different relationship matrices (Bourke et al., 2018). Here, we presented the lessons we have learned so far for implementing genomic selection in an autotetraploid and outcrossing species. We summarized previous results and also included novel findings relevant to the blueberry and polyploid community.

\subsection{Filling the gaps: phenotypic and genotypic selection in the same breeding framework}

Blueberry is an outcrossing and clonally propagated crop, for which the breeding process can be conventionally organized in two central steps: population improvement and product development (LYRENE, 2005). First, population improvement is done to manage the frequency of beneficial alleles over time by selecting and crossing outstanding materials, as conceptualized in recurrent selection designs. In parallel, product development consists of a series of trials in which potential candidates are evaluated over several years and locations, advancing across stages until the selection of the best clones becomes a registered variety. In Figure 2, we illustrated these two key steps and how they are integrated in a four-stage selection design (from Stage I to IV) in the UF blueberry breeding program.

Annually, the blueberry breeding program performs more than 200 crosses, including parents selected among cultivars, elite material, and wild germplasm (Lyrene, 2005). From these crosses, about 20,000 seedlings are planted in non-replicated high-density nurseries, establishing the so-called Stage I. After one year, plants in the Stage I are visually selected based on fruit size, color, scar, and using the breeder's 'bite test' for flavor quality attributes. Approximately $10 \%$ of the original number of seedlings are kept after this first selection. To not exhaust genetic diversity, a minimal number of individuals per family are kept. Given blueberry's long juvenile period, the availability of few berries, and the high competition in a high-density planting, it is difficult to phenotype for all traits and assess the full potential of the individuals at this stage. Additionally, the large number of individuals prevents the use of genomic prediction at this stage, given the costs of genotyping. Therefore, at Stage I, we envision that the use of marker-assisted selection (MAS) for traits with simple genetic architecture is a more feasible approach, and it is a current research line of the breeding program. In this regard, example of MAS implementation in early selection stages are reported in strawberry (GEZAN et al., 2017; Osorio et al., 2020).

After the first selection, approximately 2,000 genotypes pass to the second stage (Stage II). All plants stay in the same field plot, while unselected ones are removed. Further visual phenotypic evaluations are performed for the next 3 years. It is at this stage that we are implementing genomic prediction to increase genetic gains by improving phenotyping accuracy and selecting parents at early stages. Therefore, at Stage II, all plants will be genotyped, and the GEBVs will be predicted for five fruit quality traits (soluble solids, titratable acidity, weight, size, and firmness), yield, and consumers panel liking scores. Using a selection index according to trait importance, we will perform genomic selection to complement standard phenotypic descriptors and rank all genotypes. As routinely done, $10 \%$ of the 2,000 plants will be moved to the next stage (Stage III), where plants are clonally propagated and evaluated in a 15-plant clonal plot in a commercial field.

At Stage III, around 200 plants are more accurately phenotyped for more traits, using more fruits, clonal repetitions, and multiple years of evaluations in commercial conditions. Technically, all information collected at this stage will be used to feed the genomic prediction models. In recent years, the UF blueberry breeding program has included new traits for routine phenotyping to meet the current demand from different marketable demands. For example, the use of volatiles for flavor-assisted selection has shown the ability to predict sensory perceptions, and thus, metabolomics have been incorporated in the breeding pipeline to assess flavor ratings for a large number of genotypes (GIlBert et al., 2014; Colantonio et al., 2020; FERRÃo et al., 2020). Similarly, we want to incorporate nutraceutical compounds to leverage blueberry health benefits and shelf-life related traits so berries can withstand long periods of storage or shipment.

In the last stage (Stage IV), around 15-20 plants selected from Stage IIIs with consistent and outstanding performances are propagated and planted at commercial trial sites across the state of Florida. The different locations comprise two production systems according to the accumulation of chilling hours: evergreen and deciduous (FANG et al., 2020). To ensure accurate selection, phenotypic data is collected weekly and will 
bioRxiv preprint doi: https://doi.org/10.1101/2021.03.05.434007; this version posted March 7, 2021. The copyright holder for this preprint (which was not certified by peer review) is the author/funder, who has granted bioRxiv a license to display the preprint in perpetuity. It is made available under aCC-BY-NC-ND 4.0 International license.

also be used to feed our genomic prediction models. Fruits from selected genotypes are also submitted to sensory panels, where flavor preferences are scored by blueberry consumers. Elite selections from this final stage are ultimately named, patented, and released as clonally propagated cultivars.

Altogether, the conventional breeding pipeline takes up to 12 years to evaluate the genotype merit of an individual to be released as a cultivar. With the implementation of genomic prediction as the scope of the breeding program, the selection criteria will be more accurate than visual phenotypic selection at the Stage II. Moreover, it will shorten the time to select genotypes to advance to Stage III and to become a parent in the next breeding cycle. In a typical recurrent selection breeding scheme, parental selection is a crucial step (LyRENE, 2005). We have optimized this selection not only by ranking the GEBVs over the breeding cycles, but also by seeking crosses that minimize inbreeding. Among the different tools available for mate allocation, we have recently implemented the algorithm described in the AlphaMate software (GORJANC and HiCKEY, 2018).

\section{University of Florida \\ BLUEBERRY BREEDING PROGRAM}

Product Development Population Improvement

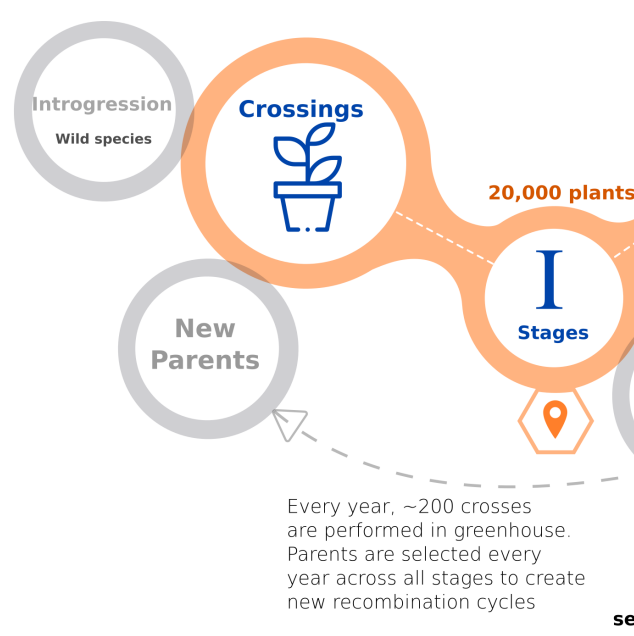

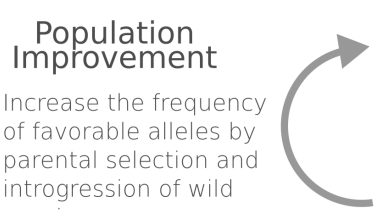
introgression of wild species
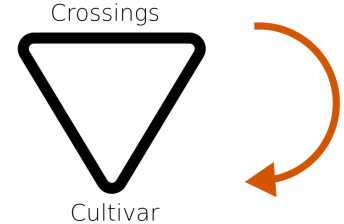

Product Development Four-stage steps from crossings to cultivar $\sim 12$ years in blueberries

Figure 2: A schematic representation of the UF blueberry breeding program, integrating phenotypic and genomic prediction. The breeding process is conventionally organized in two integrated steps: population improvement (orange) and product development (gray). A breeding cycle starts with crosses between outstanding parental genotypes. After that, several stages (I to IV) are required to evaluate the genotype performance. At Stage I, we will use marker-assisted selection targeting traits with simple genetic architecture. Genomic selection will be implemented in Stages II, when GEBVs are computed. In advanced selections (Stages III and IV), high-quality phenotyping will be performed to leverage the calibration of genomic prediction models. At these stages, the use of metabolomics and sensory panel analyses will also play an important role for flavor-assisted selections. At the end, elite materials are registered as clonally propagated cultivars. Rapid cycles could be achieved by selecting plants directly from the Stages II to Stages IV, as originally proposed by DE Bem Oliveira et al. (2019). For population improvement, over the four-stage design, elite germplasms and wild species are systematically selected to constitute new breeding cycles. 


\section{2 "Simplicity is the ultimate sophistication": on the relevance of additive GBLUP models}

When confronting the problem of modeling the relationship between molecular markers and variation in the observed traits, an important question to keep in mind is what statistical method could better describe this relationship (FERRÃO et al., 2019). In recent years, we have investigated statistical and biological aspects underlying the implementation of genomic prediction in autopolyploid species, including (i) the importance of accounting for allele dosages in whole-genome statistical models (DE BEM OliveIRA et al., 2019); (ii) the relevance of multiple gene actions, including additive and non-additive genetic sources (AMADEU et al., 2020; ZINGARETTI et al., 2020); and finally, (iii) the impact of sequencing depth of coverage, when sequence-based genotyping approaches are used (DE BEM OLIVEIRA et al., 2020).

Among the factors that differentiate diploid and polyploid analyses, resolving the allelic dosage of individual loci is one the most important. While in diploid organisms, only three genotypic classes are possible for biallelic markers; autotetraploids, like blueberry, can have up to five genotypic classes. Therefore, in theory, it is expected that statistical models accounting for the dosage effect could be more informative and provide a more realistic representation of the genetic complexity of a quantitative trait (GARCIA et al., 2013). We first tested this hypothesis by contrasting polyploid and diploid parametrizations in GWAS studies (FERRÃO et al., 2018); whereby, in fact, a larger number of associations were observed under polyploid models. In a subsequent study, we investigated a similar assumption for genomic prediction (DE BEM OLIVEIRA et al., 2019) and tested GBLUP models using relationship matrices built in a tetraploid (SLATER et al., 2016) and diploid (VANRADEN, 2008) fashion. Interestingly, both parametrizations resulted in similar performances for all traits tested. Similar predictive ability for diploid and polyploid parametrizations were also reported in other autotetraploid species (LARA et al., 2019; Matias et al., 2019), which ultimately reinforced the robustness of the predictive capacity of GBLUP regardless of the ploidy parametrization used.

Besides the potential additive impact of allele dosages, dominance effects can also be heritable in polyploids and could improve the prediction of genetic values. Therefore, it is also reasonable to speculate that a greater number of alleles per locus may increase the range of genetic models to describe one-locus genotypic value by accounting for multiple dominance levels (GALLAIS, 2003). This is exemplified by the different models addressing the dominance effect proposed in the polyploid literature, including the use of digenic interactions (ENDELMAN et al., 2018), the use of a general effect by assuming that each genotype has its own effect (Rosyara et al., 2016; Slater et al., 2016), and the use of heterozygous parametrization (ENCisORODRIGUEZ et al., 2018). In blueberries, we tested the importance of such different gene action in predictive studies. Although we have observed an improvement in the statistical goodness-of-fit when dominance effects are counted, this increment is not directly translated into predictive ability (AMADEU et al., 2020). Hence, the additive model resulted in performance similar to models accounting for dominance effects, as it has been described for diploid species (MUÑOz et al., 2014).

Given the genetic complexity of polyploids and the potentially higher levels of intra- and inter-locus interactions, we also hypothesized that predictions could be improved by using deep learning techniques (ZingaretTi et al., 2020). Through deep learning, we could take advantage of non-linearity assumptions to model the whole genetic merit of an individual. To test this, we used allo-octoploid strawberry and autotetraploid blueberry as our biological models and compared linear models and deep learning techniques for prediction. In both species, we did not observe improvements of deep learning over traditional linear models for traits with presumably different genetic architectures. The only exception was observed in a simulated data set, in which deep learning performed better for traits with large epistatic effects and low narrow-sense heritability. This again, reinforced the high predictive capacity of mixed models as prediction machinery.

Our last contribution for the practical implementation of genomic prediction in polyploids is regarding the relevance of sequencing depth of coverage for genotyping methods based on next-generation sequencing. Sequencing depth refers to the number of reads sequenced at a given site in the genome. Low coverage datasets increase the chances of not sampling all homologous chromosomes at a given site for a given indi-

\footnotetext{
${ }^{1}$ Quote by Leonardo da Vinci
} 
Table 1: Predictive ability between two different genotype calling approaches (dosage and ratio) under two sequencing depth scenarios (6x and 60x) for five fruit quality traits in blueberry using 10-fold cross-validation. Results are means and, between brackets, the range observed.

\begin{tabular}{llllll}
\hline Method & Depth & Firmness & Size & Weight & Brix \\
\hline Dosage & $6 \mathrm{x}$ & $0.44[0.40-0.52]$ & $0.38[0.29-0.49]$ & $0.47[0.40-0.57]$ & $0.28[0.18-0.47]$ \\
Dosage & $60 \mathrm{x}$ & $0.46[0.41-0.55]$ & $0.40[0.29-0.53]$ & $0.49[0.40-0.56]$ & $0.29[0.17-0.46]$ \\
Ratio & $6 \mathrm{x}$ & $0.45[0.38-0.55]$ & $0.40[0.31-0.48]$ & $0.48[0.42-0.56]$ & $0.28[0.18-0.43]$ \\
Ratio & $60 \mathrm{x}$ & $0.46[0.40-0.54]$ & $0.39[0.29-0.51]$ & $0.49[0.41-0.56]$ & $0.29[0.17-0.47]$ \\
\hline
\end{tabular}

vidual during sequencing. It could result in high rates of missing data, miscalled genotypes, and uncertainty of allele copy number in heterozygous genotypes (ClARK et al., 2019). To circumvent this issue, some studies in polyploid crops have recommended increasing the sequencing depth, which implies higher costs of genotyping. For example, BAstien et al. (2018) and Uitdewilligen et al. (2013) suggested sequencing depths of 50X-80X for an accurate assessment of allele dosage in autotetraploid potatoes. In a recent study, we demonstrated that such numbers are quite conservative for genomic prediction. By combining a simple genetic parametrization (ratio) and low-to-mid sequencing depth $(6 \mathrm{x}-12 \mathrm{x})$, we achieved similar predictive accuracies as higher-depths for blueberry traits with different genetic architectures (DE BEM OLIVEIRA et al., 2020). Similar results are also reported by ZHeng et al. (2020). In practical terms, reducing the amount of sequencing data will also reduce the costs associated with implementing genomic selection or potentially genotyping more individuals under a fixed budget.

Despite the considerable advancements previously explored, the relevance of using more sophisticated algorithms for genotype calling and its impact on genomic prediction remains unexplored. Recently, several new methods have been developed to assign accurate allelic dosage of individual loci in polyploids (GARCIA et al., 2013; Gerard et al., 2018; Pereira et al., 2018; Clark et al., 2019). In this paper, we compared predictive abilities using different genomic parametrization and confirmed that low-to-mid sequencing depth and ratio parametrization can be used for ranking GEBVs - with similar predictive performance (Table 1) and genotypic ranking (Table 2). Nonetheless, despite the attractive simplicity of using the ratio and low-sequencing depth, such results are only valid for prediction studies (DE BEM OLIVEIRA et al., 2019, 2020). Importantly, there is no empirical evidence that setting the parameters to these levels could work for inferential studies such as GWAS, population genomics, linkage and QTL mapping. In this sense, an important counterpoint was recently reported in hexaploid sweet potato, for which higher sequencing depths and accurate dosage calling improved the ultra-dense linkage map and posterior QTL analysis (GEMENET et al., 2020; MolLinari et al., 2020). For GWAS, we observed large rates of false positive associations when analyses were performed using low sequencing depth associated to the ratio parametrization (results not shown). Herein, we systematically observed large biases when relationship matrices were constructed using the ratio_6x approach (Figure S1 and Table S2).

In summary, our results are suggesting that the use of traditional GBLUP is robust enough for genomic prediction, even under the simplistic assumptions. This fact that has long been discussed in the specialized literature, and has raised questions on the contribution of linkage disequilibrium between QTLs and markers versus the relationship information to genomic selection (HABIER et al., 2013).

\subsection{How does genomic prediction work in a real validation population?}

While we have investigated several statistical and computational aspects related to genomic selection in blueberry, it is still unknown how accurate the predictions will be across breeding cycles, with plants in different phenological stages and locations. This scenario came to be called "true validation" and involves the use of independent populations. We investigate it by dividing our prediction analyses as following: models calibrated in 2014 and 2015 using plants in Stage II were used for phenotypic predictions of Stages 
Table 2: Number of clones matching the top 20 ranking of clones using the dosage_60x method as benchmark, under 10-folds cross-validation. A post hoc Tukey test (alpha=.05) was used for intergroup comparisons over the scenarios. Cells with the same letter represent non-statistically different groups for the given trait (column).

\begin{tabular}{llllll}
\hline Method & Depth & Firmness & Size & Weight & Brix \\
\hline Dosage & $6 \mathrm{x}$ & $16.5^{b}$ & $16.9^{b}$ & $16.3^{b}$ & $16.4 \mathrm{~b}$ \\
Ratio & $6 \mathrm{x}$ & $16.2^{b}$ & $15.6^{c}$ & $16.2^{b}$ & $15.3 \mathrm{~b}$ \\
Ratio & $60 \mathrm{x}$ & $18.8^{a}$ & $18.3^{a}$ & $18.6^{a}$ & $18.7 \mathrm{a}$ \\
\hline
\end{tabular}

III and IV individuals. Both data sets share genetic similarity (Figure 3 a).

For true validations, we tested different scenarios in which genomic selection could be applied (Figure 1). First, we predicted the overall performance using genomic and pedigree information and confirmed the importance of genomic information (general predictions, in Figure 3b). When compared to predictions using within-sample cross-validation schemes, as reported in DE BEM OliveIRA et al. (2019) and AMADEU et al. (2020), we observed a reduction on the predictive results (Table S1). This decline in predictive performance in real validation is expected, due to differences in the allele frequencies over populations, variation in linkage disequilibrium patterns, and genotype-by-environment interactions (HABIER et al., 2013).

Another predictive scenario focused on validations across breeding cycles. To this end, we used the calibration test - originally evaluated in Stages II - to predict a subgroup of individuals that were cloned and planted in an advanced stage (Stages III). On average, larger values were observed compared to the general scenario, but it was still, as expected, lower than within-sample cross-validation schemes (acrossstages predictions, Figure 3c). These results highlight (i) the importance of collecting better phenotypic data and (ii) the influence of the plant management. Remarkably, most of the phenotypic traits measured in the calibration set were collected from five berries per genotype; while on Stage III, we used 25 berries per genotype. Furthermore, genotypes in Stage II are planted in high-density nurseries with phenotypes collected in plants that are still in their juvenile phase, while Stages III are grown under commercial conditions.

In the third scenario, a more challenging exercise was to measure how predictive capacity varies across regions in the State of Florida (stratified predictions, Figure 3d). Higher predictability was observed for Citra and Central-FL, the closest regions where the models were originally trained. In counterpart, plants evaluated in the South-FL showed, on average, lower predictability performances. Despite the small number of genotypes included in this analysis, these results provide insights into the importance of genotype-byenvironment $(\mathrm{GxE})$ interaction for genomic selection in blueberry. We further explored this hypothesis by using a group of 16 common genotypes (checks) evaluated over the four regions. The results confirmed the significance of the GxE effect for most of the traits (Table 3), with the plants evaluated in South-FL showing the most contrasting values. It is noteworthy that blueberry locations in South-FL are grown under an evergreen production system, under less chilling hours, and are focused on preventing defoliation during the winter months (FANG et al., 2020). On the other hand, the location in Citra, Central-FL, and North-FL regions are grown under the deciduous production system, where leaves are dropped during the winter. Such differences in the production systems could be driving the largest disparity observed at South-FL, when compared to the other regions.

The results from real validations allow us to draw some practical conclusions. First, even with lowto-moderate predictive accuracies, genomic selection is still encouraging. For example, soluble solids and firmness are both traits treasured by consumers, for which routine phenotyping is expensive and timeconsuming for large populations, like Stage IIs. Ranking plants based on their GEBVs proved to be a better alternative than any other criteria historically used over the course of UF blueberry breeding program (pedigree or visual selection). More accurate phenotypic data to annually re-calibrate the model has also the potential to improve predictability. Finally, we also reinforce the importance to recalibrate our models considering the environment targets. With the recent advent of high-throughput phenotyping, we envision 
Table 3: Mean and standard deviation (in parenthesis) of four fruit quality traits evaluated in advanced stages of the blueberry breeding program at four regions of Florida. Values were computed using 16 common genotypes (checks).

\begin{tabular}{lllll}
\hline Location & Firmness & Size & Weight & Brix \\
\hline North FL & $248(32.8)$ & $18.0(1.79)$ & $2.57(0.641)$ & $11.3(1.31)$ \\
Citra FL & $245(42.0)$ & $17.0(2.22)$ & $2.34(0.691)$ & $10.9(1.33)$ \\
Central FL & $244(29.3)$ & $17.7(1.46)$ & $2.29(0.491)$ & $11.8(1.27)$ \\
South FL & $251(33.4)$ & $17.4(1.34)$ & $2.21(0.549)$ & $12.0(1.91)$ \\
\hline GxE (p.value)* & 0.007 & 0.0002 & 0.005 & 0.47 \\
\hline
\end{tabular}

* P.values associated to genotype-by-environment interaction $(\mathrm{GxE})$ were computed using a linear model and Analysis of Variance (ANOVA), where season, genotype, location, and the interaction between genotype and location (GxE) were fitted as fixed effects.

that more data across different production systems in Florida - and around the globe- could be used for better calibration and ultimately, more accurate predictions. Examples of image analyses for high-throughput phenotyping have been reported in other fruit trees (Diaz-Garcia et al., 2016; Di Gennaro et al., 2019; Koirala et al., 2019; Feldmann et al., 2020), including blueberries (Jiang et al., 2019).

\subsection{Unifying biological discoveries and predictions}

The use of genomics information also provides new opportunities to integrate biotechnology and quantitative genetics into modern breeding programs, creating platforms for both delivery of new products and biological discovery (HICKEY et al., 2017). In blueberry, biological discoveries have been addressed via QTL mapping (CAPPAi et al., 2020a) and GWAS studies (FERRÃo et al., 2019, 2020) for multiple fruit quality traits. Unifying such new discoveries with prediction is challenging, but it has been addressed under three different avenues: (i) use of GWAS discovered QTLs as fixed effects on GS models; (ii) incorporating markers (or QTL) in MAS designs; and (iii) using genome-editing technology to speed up breeding.

In a strategy called "GS de novo GWAS", we explored the importance and applicability of GWAS findings for prediction by using the significant GWAS hits as fixed effects in GS models, considering independent datasets. For oligogenic traits, like some flavor-related volatiles, we achieved an increase of more than $20 \%$ in the predictive ability, when compared with traditional GS methods (FERRÃo et al., 2018). Using a similar strategy, gains in predictive performance have been also reported in other crops, such as maize (BERNARDO, 2014) (RICE and LiPKA, 2019), wheat (SEHGAL et al., 2020), and rice (SPINDEL et al., 2016). Alternatively, we have investigated further modelling strategies to accommodate biological information into the predictive models. For example, the use of Bayesian strategies that could accommodate SNPs with larger effect by using different prior distributions (Gianola, 2013; Zhou et al., 2013; ErBe et al., 2012); and GBLUP models that could weight variants previously selected either via association analysis or using bioinformatic pipelines (Su et al., 2014; Zhang et al., 2016; Liu et al., 2020; Ren et al., 2021).

Another potential strategy is to use target markers associated with important traits for MAS during Stage I of the blueberry breeding program. Such a strategy could be used for early selection of plants still in the seedling stage. Acknowledged by their simple genetic architecture, we showed that few markers could yield reasonable predictive accuracies of volatile emission and, thus, leverage flavor selection (FERRÃO et al., 2020). We envision that MAS can also be implemented for other oligogenic traits. In this regard, we have been conducting other GWAS and QTL mapping studies for disease resistance, such as anthracnose (Colletotrichum gloeosporioides) and bacterial wilt (Ralstonia solanacearum). A similar strategy has been implemented in strawberry (GEZAN et al., 2017; Osorio et al., 2020) and other fruits (IEZZONI et al., 2020). However, for MAS to be applicable for thousands of plants, fast DNA extraction and SNP genotyping assays should be optimized.

Gene editing is another attractive technology with potential to have significant effects in the breeding program. Aside from the use of CRISPR-Cas9 for validating candidate genes identified via GWAS or QTL 
bioRxiv preprint doi: https://doi.org/10.1101/2021.03.05.434007; this version posted March 7, 2021. The copyright holder for this preprint (which was not certified by peer review) is the author/funder, who has granted bioRxiv a license to display the preprint in perpetuity. It is made available under aCC-BY-NC-ND 4.0 International license.

(A)

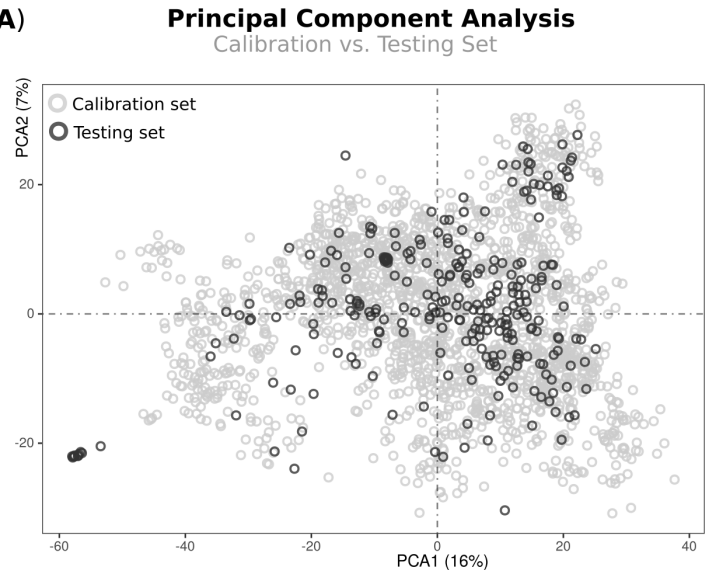

(D)

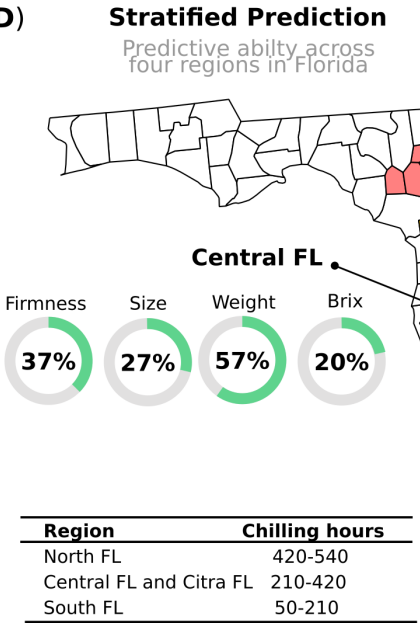

(B)

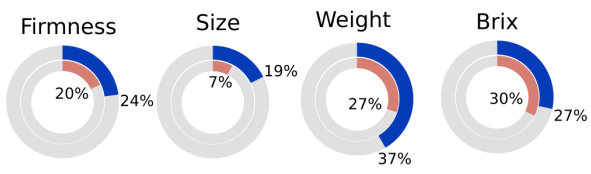

(C)

Across-stages predictions

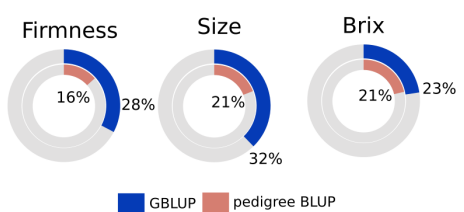

$25 \%{ }_{15 \%} 23 \% \quad{ }_{15 \%}^{\text {Wize }}$

Citra FL* Firmness Size Weight Brix

$42 \% \quad 46 \%$

$20 \%$

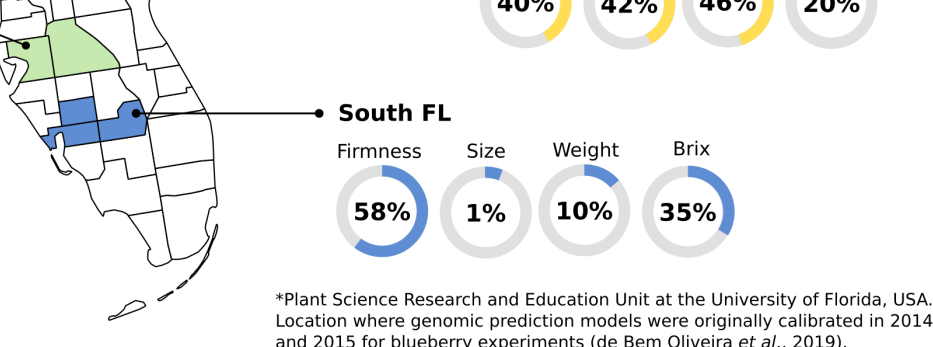

Figure 3: Phenotypic prediction. (A) Principal component analysis (PCA) of two blueberry populations: calibration set represents the trained set, where genomic prediction models were originally calibrated, and testing set comprising additional 280 individuals used for testing. (B) General prediction: predictive ability of the testing set, after training the models in the calibration set. (C) Across-stages prediction: predictive ability measured in a group of 114 individuals that was also included in the calibration set, but phenotypes were collected in advanced selection stages. (D) Stratified predictions: after training the models in the calibration set, individuals in the testing set were predicted using phenotypes collected over four macro-regions in the Florida State, which are under different chilling hour accumulation.

studies, some simulations have recently shown that genome editing can double the rate of genomic gain when coupled with genomic prediction, compared to genomic selection conducted in isolation (NomAN et al., 2016; HiCKEY et al., 2017). To our knowledge, there is only one study of CRISPR-Cas9 targeted mutagenesis in blueberry (OMORI et al., 2021). At the UF blueberry breeding program, we have advanced our understanding on the best tissue culture practices and most effective transformation markers (CAPPAI et al., 2020b), laying the ground for CRISPR/Cas9 genome editing implementation in our breeding program. Using this technique, we can also take advantage of the knowledge accumulated from model crops to introduce novel allelic diversity in orthologues and accelerate the domestication process. 


\section{Conclusions ans Future Directions}

The implementation of genomic selection has already changed the UF blueberry breeding program routine, by reorganizing the way we collect genotype and phenotype information and analyze data to rank the material to advance stages and to breed in the next cycles. Our previous studies on genomic selection were fundamental to define the most cost- and time-effective methods for model parameterization and genotyping. The main lessons learned can be conveniently divided in different areas. Statistically, despite the numerous algorithms for prediction - many of them more elegant at the biological and computational level - it was the use of additive effects under a linear mixed model framework (GBLUP) that showed the best balance between efficiency and accuracy. Considering the particularities of autopolyploid genetic data, we showed that for genomic selection, low depth of sequencing $(6 \mathrm{x}-12 \mathrm{x})$ and simplifying the allele dosage information (i.e., diploidization and ratio) resulted in similar prediction accuracies as those obtained using more refined scenarios. At the practical level, genomic prediction was incorporated in a recurrent selection breeding scheme, whereby variety deployment and populational improvement run in parallel. So far, GEBVs have been primarily used for parental selection to increase genetic gains, while keeping the genetic diversity.

Finally, we highlight some challenges and potential opportunities for further studies in blueberries. First, re-calibrating the model with more accurate phenotypic data can yield better predictive ability. In this sense, phenomics is also a cutting-edge area of research that could leverage the number of samples collected during a season and improve the quality of phenotypic data. For example, yield is a complex and timeconsuming trait to be phenotyped over the season. We envision that image-based phenotyping may aid on the task of evaluating yield and other traits associated to plant architecture and diseases. Statistically, testing new algorithms for mate allocation, and using haplotypes for prediction and imputation methods are some potential areas that could further improve genomic predictions.

\section{Conflict of Interest}

The authors declare that the research was conducted in the absence of any commercial or financial relationships that could be construed as a potential conflict of interest.

\section{Acknowledgements}

The authors thank Catherine Cellon and James Olmstead for the calibration set data collection; Werner Collante, Lauren Scott, and Douglas Phillips for the testing set data collection; and Mia Acker for reviewing the manuscript. This work was supported by the UF royalty fund generated by the licensing of blueberry cultivars.

\section{Author contributions}

PRM and LFVF conceived and supervised the study. JB coordinated the collection and genotyping of the samples. IBO coordinated the collection the data for real validation. LFVF and RRA analyzed and interpreted the phenotypic and genomic selection results. LFVF wrote the paper and included the revision from all authors. All authors read and approved the final version of the manuscript for publication.

\section{References}

Amadeu, R. R., C. Cellon, J. W. Olmstead, A. A. F. Garcia, M. F. R. Resende, and P. R. MuÑoz, 2016 AGHmatrix: R Package to Construct Relationship Matrices for Autotetraploid and Diploid Species: A Blueberry Example. The Plant Genome 9: 0.

Amadeu, R. R., L. F. V. Ferrão, I. D. B. Oliveira, J. Benevenuto, J. B. Endelman, and P. R. MunOz, 2020 Impact of dominance effects on autotetraploid genomic prediction. Crop Science 60: 656665. 
Bastien, M., C. Boudhrioua, G. Fortin, and F. Belzile, 2018 Exploring the potential and limitations of genotyping-by-sequencing for SNP discovery and genotyping in tetraploid potato. Genome 61: 449-456.

Benevenuto, J., L. F. V. Ferrão, R. R. Amadeu, and P. Munoz, 2019 How can a high-quality genome assembly help plant breeders? GigaScience 8: giz068.

Bernardo, R., 2014 Genomewide Selection when Major Genes Are Known. Crop Science 54.

Bourke, P. M., R. E. Voorrips, R. G. F. Visser, and C. Maliepaard, 2018 Tools for genetic studies in experimental populations of polyploids. Frontiers in plant science 9: 513.

Butler, D., B. R. Cullis, A. Gilmour, and B. Gogel, 2009 Asreml-r reference manual. The State of Queensland, Department of Primary Industries and Fisheries, Brisbane .

Cappai, F., R. R. Amadeu, J. Benevenuto, R. Cullen, A. Garcia, A. Grossman, L. F. V. FerRão, and P. Munoz, 2020a High-Resolution Linkage Map and QTL Analyses of Fruit Firmness in Autotetraploid Blueberry. Frontiers in plant science 11: 1767.

Cappai, F., A. Garcia, R. Cullen, M. Davis, and P. R. Munoz, 2020b Advancements in Low-Chill Blueberry Vaccinium corymbosum L. Tissue Culture Practices. Plants 9: 1624.

Caruana, B. M., L. W. Pembleton, F. Constable, B. Rodoni, A. T. Slater, and N. O. I. Cogan, 2019 Validation of genotyping by sequencing using transcriptomics for diversity and application of genomic selection in tetraploid potato. Frontiers in plant science 10: 670 .

Cellon, C., R. R. Amadeu, J. W. Olmstead, M. R. Mattia, L. F. V. Ferrao, and P. R. Munoz, 2018 Estimation of genetic parameters and prediction of breeding values in an autotetraploid blueberry breeding population with extensive pedigree data. Euphytica 214: 1-13.

Clark, L. V., A. E. LiPka, and E. J. SACKs, 2019 polyRAD: Genotype calling with uncertainty from sequencing data in polyploids and diploids. G3: Genes, Genomes, Genetics 9: 663-673.

Colantonio, V., L. F. V. Ferrao, D. Tieman, N. Bliznyuk, C. Sims, H. Klee, P. R. Munoz, and M. F. R. REsende, 2020 Metabolomic Selection for Enhanced Fruit Flavor. bioRxiv .

Colle, M., C. P. Leisner, C. M. Wai, S. Ou, K. A. Bird, J. Wang, J. H. Wisecaver, A. E. Yocca, E. I. Alger, and H. TANG, 2019 Haplotype-phased genome and evolution of phytonutrient pathways of tetraploid blueberry. GigaScience 8: giz012.

Danecek, P., A. Auton, G. Abecasis, C. A. Albers, E. Banks, M. A. DePristo, R. E. Handsaker, G. Lunter, G. T. Marth, S. T. Sherry, G. McVean, R. Durbin, and . G. P. A. Group, 2011 The variant call format and VCFtools. Bioinformatics 27: 2156-2158.

de Bem Oliveira, I., R. R. Amadeu, L. F. V. Ferrão, and P. R. Muñoz, 2020 Optimizing wholegenomic prediction for autotetraploid blueberry breeding. Heredity 125: 437-448.

de Bem Oliveira, I., M. F. R. Resende, L. F. V. Ferrão, R. R. Amadeu, J. B. Endelman, M. Kirst, A. S. G. Coelho, and P. R. Munoz, 2019 Genomic prediction of autotetraploids; influence of relationship matrices, allele dosage, and continuous genotyping calls in phenotype prediction. G3: Genes, Genomes, Genetics 9: 1189-1198.

Di Gennaro, S. F., P. Toscano, P. Cinat, A. Berton, and A. Matese, 2019 A low-cost and unsupervised image recognition methodology for yield estimation in a vineyard. Frontiers in plant science 10: 559.

Diaz-Garcia, L., G. Covarrubias-Pazaran, B. Schlautman, and J. Zalapa, 2016 GinA, an efficient and high-throughput software for horticultural phenotyping. PloS one 11: e0160439. 
Dufresne, F., M. Stift, R. Vergilino, and B. K. Mable, 2014 Recent progress and challenges in population genetics of polyploid organisms: an overview of current state-of-the-art molecular and statistical tools. Molecular ecology 23: 40-69.

Enciso-Rodriguez, F., D. Douches, M. Lopez-Cruz, J. Coombs, and G. De Los Campos, 2018 Genomic selection for late blight and common scab resistance in tetraploid potato (Solanum tuberosum). G3: Genes, Genomes, Genetics 8: 2471-2481.

Endelman, J. B., 2011 Ridge regression and other kernels for genomic selection with R package rrBLUP. The plant genome 4 .

Endelman, J. B., C. A. S. Carley, P. C. Bethre, J. J. Coombs, M. E. Clough, W. L. da Silva, W. S. De Jong, D. S. Douches, C. M. Frederick, and K. G. Haynes, 2018 Genetic variance partitioning and genome-wide prediction with allele dosage information in autotetraploid potato. Genetics 209: $77-87$.

Erbe, M., B. Hayes, L. Matukumalli, S. Goswami, P. Bowman, C. Reich, B. Mason, and M. GodDARD, 2012 Improving accuracy of genomic predictions within and between dairy cattle breeds with imputed high-density single nucleotide polymorphism panels. Journal of dairy science 95: 4114-4129.

Fang, Y., G. H. Nunez, M. N. Da Silva, D. A. Phillips, and P. R. Munoz, 2020 A Review for Southern Highbush Blueberry Alternative Production Systems. Agronomy 10: 1531.

FAOSTAT, 2021 FAOSTAT.

Feldmann, M. J., M. A. Hardigan, R. A. Famula, C. M. Lopez, A. Tabb, G. S. Cole, and S. J. KNAPP, 2020 Multi-dimensional machine learning approaches for fruit shape phenotyping in strawberry. GigaScience 9: giaa030.

Ferrão, L. F. V., J. Benevenuto, I. D. B. Oliveira, C. Cellon, J. Olmstead, M. Kirst, M. F. REsende JR, and P. R. Munoz, 2018 Insights into the genetic basis of blueberry fruit-related traits using diploid and polyploid models in a GWAS context. Frontiers in Ecology and Evolution 6: 107.

Ferrão, L. F. V., R. G. Ferrão, M. A. G. Ferrão, A. Fonseca, P. Carbonetto, M. Stephens, and A. A. F. GARCIA, 2019 Accurate genomic prediction of Coffea canephora in multiple environments using whole-genome statistical models. Heredity 122: 261-275.

Ferrão, L. F. V., T. S. Johnson, J. Benevenuto, P. P. Edger, T. A. Colquhoun, and P. R. Munoz, 2020 Genome-wide association of volatiles reveals candidate loci for blueberry flavor. New Phytologist 226: 1725-1737.

GaLlais, A., 2003 Quantitative genetics and breeding methods in autopolyploid plants. Quae.

Garcia, A. A. F., M. Mollinari, T. G. Marconi, O. R. Serang, R. R. Silva, M. L. C. Vieira, R. Vicentini, E. A. Costa, M. C. Mancini, and M. O. S. Garcia, 2013 SNP genotyping allows an in-depth characterisation of the genome of sugarcane and other complex autopolyploids. Scientific reports 3.

Garrison, E. and G. Marth, 2012 Haplotype-based variant detection from short-read sequencing. arXiv preprint arXiv:1207.3907 .

Gemenet, D. C., G. da Silva Pereira, B. De Boeck, J. C. Wood, M. Mollinari, B. A. Olukolu, F. Diaz, V. Mosquera, R. T. Ssali, and M. David, 2020 Quantitative trait loci and differential gene expression analyses reveal the genetic basis for negatively associated $\beta$-carotene and starch content in hexaploid sweetpotato [Ipomoea batatas (L.) Lam.]. Theoretical and Applied Genetics 133: 23-36.

Gerard, D. and L. F. V. Ferrão, 2020 Priors for genotyping polyploids. Bioinformatics 36: 1795-1800. 
Gerard, D., L. F. V. Ferrão, A. A. F. Garcia, and M. Stephens, 2018 Genotyping polyploids from messy sequencing data. Genetics 210: 789-807.

Gezan, S. A., L. F. Osorio, S. Verma, and V. M. Whitaker, 2017 An experimental validation of genomic selection in octoploid strawberry. Horticulture research 4: 1-9.

Gianola, D., 2013 Priors in Whole-Genome Regression: The Bayesian Alphabet Returns 194: 573-596.

Gilbert, J. L., J. W. Olmstead, T. A. Colquhoun, L. A. Levin, D. G. Clark, and H. R. Moskowitz, 2014 Consumer-assisted selection of blueberry fruit quality traits. HortScience 49: 864873.

GorJanc, G. and J. M. Hickey, 2018 AlphaMate: a program for optimizing selection, maintenance of diversity and mate allocation in breeding programs. Bioinformatics 34: 3408-3411.

Habier, D., R. L. Fernando, and D. J. Garrick, 2013 Genomic BLUP decoded: a look into the black box of genomic prediction. Genetics 194: 597-607.

Hickey, J. M., T. Chiurugwi, I. Mackay, W. Powell, A. Eggen, A. Kilian, C. Jones, C. Canales, D. Grattapaglia, and F. Bassi, 2017 Genomic prediction unifies animal and plant breeding programs to form platforms for biological discovery. Nature genetics 49: 1297.

Iezzoni, A. F., J. McFerson, J. Luby, K. Gasic, V. Whitaker, N. Bassil, C. Yue, K. Gallardo, V. MCCRACKen, and M. Coe, 2020 RosBREED: bridging the chasm between discovery and application to enable DNA-informed breeding in rosaceous crops. Horticulture research 7: 1-23.

Jiang, Y., C. Li, F. Takeda, E. A. Kramer, H. Ashrafi, and J. Hunter, 2019 3D point cloud data to quantitatively characterize size and shape of shrub crops. Horticulture research 6: 1-17.

Kalt, W., A. Cassidy, L. R. Howard, R. Krikorian, A. J. Stull, F. Tremblay, and R. ZamoraRos, 2020 Recent research on the health benefits of blueberries and their anthocyanins. Advances in Nutrition 11: 224-236.

Koirala, A., K. B. Walsh, Z. Wang, and C. McCarthy, 2019 Deep learning-Method overview and review of use for fruit detection and yield estimation. Computers and electronics in agriculture 162: $219-234$.

Lara, L. A. D. C., M. F. Santos, L. Jank, L. Chiari, M. D. M. Vilela, R. R. Amadeu, J. P. R. dos Santos, G. D. S. Pereira, Z.-B. Zeng, and A. A. F. Garcia, 2019 Genomic Selection with Allele Dosage in Panicum maximum Jacq. G3: Genes, Genomes, Genetics 9: 2463-2475.

Lee, W.-P., M. P. Stromberg, A. Ward, C. Stewart, E. P. Garrison, and G. T. Marth, 2014 MOSAIK: a hash-based algorithm for accurate next-generation sequencing short-read mapping. PloS one 9: e90581.

Liu, A., M. S. Lund, D. Boichard, E. Karaman, B. Guldbrandtsen, S. Fritz, G. P. Aamand, U. S. Nielsen, G. Sahana, and Y. Wang, 2020 Weighted single-step genomic best linear unbiased prediction integrating variants selected from sequencing data by association and bioinformatics analyses. Genetics Selection Evolution 52: 1-17.

Lyrene, P. M., 2000 Breeding southern highbush blueberries in Florida. In VII International Symposium on Vaccinium Culture 574, pp. 149-152.

Lyrene, P. M., 2005 Breeding low-chill blueberries and peaches for subtropical areas. HortScience 40: $1947-1949$.

Mackay, I., H. Piepho, and A. A. F. Garcia, 2019 Statistical methods for plant breeding. Handbook of Statistical Genomics: Two Volume Set pp. 501-520. 
Matias, F. I., F. C. Alves, K. G. X. Meireles, S. C. L. Barrios, C. B. do Valle, J. B. Endelman, and R. FRITSChe-Neto, 2019 On the accuracy of genomic prediction models considering multi-trait and allele dosage in Urochloa spp. interspecific tetraploid hybrids. Molecular Breeding 39: 100.

Meuwissen, T. H., B. J. Hayes, and M. E. Goddard, 2001 Prediction of total genetic value using genome-wide dense marker maps. Genetics 157: 1819-1829.

Mollinari, M., B. A. Olukolu, G. D. S. Pereira, A. Khan, D. Gemenet, G. C. Yencho, and Z.-B. ZENG, 2020 Unraveling the hexaploid sweetpotato inheritance using ultra-dense multilocus mapping. G3: Genes, Genomes, Genetics 10: 281-292.

Muñoz, P. R., M. F. R. Resende, S. A. Gezan, M. D. V. Resende, G. de los Campos, M. Kirst, D. Huber, and G. F. Peter, 2014 Unraveling additive from nonadditive effects using genomic relationship matrices. Genetics 198: 1759-1768.

Noman, A., M. Aqeel, and S. He, 2016 CRISPR-Cas9: tool for qualitative and quantitative plant genome editing. Frontiers in plant science 7: 1740.

Omori, M., H. Yamane, K. Osakabe, Y. Osakabe, and R. Tao, 2021 Targeted mutagenesis of CENTRORADIALIS using CRISPR/Cas9 system through the improvement of genetic transformation efficiency of tetraploid highbush blueberry. The Journal of Horticultural Science and Biotechnology 96: 153-161.

Osorio, L. F., S. A. Gezan, S. Verma, and V. Whitaker, 2020 Independent validation of genomic prediction in strawberry over multiple cycles. Frontiers in Genetics 11: 1862.

Pereira, G. S., A. A. F. Garcia, and G. R. A. Margarido, 2018 A fully automated pipeline for quantitative genotype calling from next generation sequencing data in autopolyploids. BMC bioinformatics 19: $1-10$.

Pritchard, J. K., M. Stephens, N. A. Rosenberg, and P. Donnelly, 2000 Association Mapping in Structured Populations. The American Journal of Human Genetics 67: 170-181.

Ren, D., L. An, B. Li, L. Qiao, and W. Liu, 2021 Efficient weighting methods for genomic best linearunbiased prediction (blup) adapted to the genetic architectures of quantitative traits. Heredity 126: $320-$ 334.

Rice, B. and A. E. LIPKA, 2019 Evaluation of RR-BLUP Genomic Selection Models that Incorporate Peak Genome-Wide Association Study Signals in Maize and Sorghum. The Plant Genome 12: 180052.

Rosyara, U. R., W. S. De Jong, D. S. Douches, and J. B. Endelman, 2016 Software for genome-wide association studies in autopolyploids and its application to potato. The plant genome $\mathbf{9}$.

Sehgal, D., U. Rosyara, S. Mondal, R. Singh, J. Poland, and S. Dreisigacker, 2020 Incorporating genome-wide association mapping results into genomic prediction models for grain yield and yield stability in CIMMYT spring bread wheat. Frontiers in plant science 11: 197.

Sharpe, R. H. and W. B. Sherman, 1971 Breeding blueberries for low-chilling requirement. HortScience

Slater, A. T., N. O. I. Cogan, J. W. Forster, B. J. Hayes, and H. D. Daetwyler, 2016 Improving genetic gain with genomic selection in autotetraploid potato. The plant genome $\mathbf{9}$.

Spindel, J. E., H. Begum, D. Akdemir, B. Collard, E. Redoña, J.-L. Jannink, and S. Mccouch, 2016 Genome-wide prediction models that incorporate de novo GWAS are a powerful new tool for tropical rice improvement. Heredity 116: 395-408. 
Su, G., O. F. Christensen, L. Janss, and M. S. Lund, 2014 Comparison of genomic predictions using genomic relationship matrices built with different weighting factors to account for locus-specific variances. Journal of dairy science 97: 6547-6559.

Sverrisdóttir, E., S. Byrne, E. H. R. Sundmark, H. Ø. Johnsen, H. G. Kirk, T. Asp, L. Janss, and K. L. Nielsen, 2017 Genomic prediction of starch content and chipping quality in tetraploid potato using genotyping-by-sequencing. Theoretical and Applied Genetics 130: 2091-2108.

TEAm, R. C., 2013 R: A language and environment for statistical computing .

Uitdewilligen, J. G., A.-M. A. Wolters, B. Bjorn, T. J. A. Borm, R. G. F. Visser, and H. J. VAN ECK, 2013 A next-generation sequencing method for genotyping-by-sequencing of highly heterozygous autotetraploid potato. PLoS One 8: e62355.

VAnRaden, P. M., 2008 Efficient methods to compute genomic predictions. Journal of dairy science 91: 4414-4423.

Zhang, X., D. Lourenco, I. Aguilar, A. Legarra, and I. Misztal, 2016 Weighting strategies for single-step genomic BLUP: an iterative approach for accurate calculation of GEBV and GWAS. Frontiers in genetics 7: 151.

Zheng, C., R. R. Amadeu, P. Munoz, and J. B. Endelman, 2020 Haplotype reconstruction in connected tetraploid F1 populations. bioRxiv .

Zhou, X., P. Carbonetto, and M. Stephens, 2013 Polygenic modeling with bayesian sparse linear mixed models. PLoS Genet 9: e1003264.

Zingaretti, L. M., S. A. Gezan, L. F. V. Ferrão, L. F. Osorio, A. Monfort, P. R. Muñoz, V. M. Whitaker, and M. PÉREz-Enciso, 2020 Exploring deep learning for complex trait genomic prediction in polyploid outcrossing species. Frontiers in plant science 11: 25. 